\title{
OPERATORS ON BANACH SPACES TAKING COMPACT SETS INSIDE RANGES OF VECTOR MEASURES
}

\author{
CÁNDIDO PIÑEIRO
}

\author{
(Communicated by Palle E. T. Jorgensen)
}

\begin{abstract}
Let $X$ and $Y$ be real Banach spaces. We prove that an operator $T$ from $X$ into $Y$ maps compact subsets of $X$ into subsets of $Y$ that lie inside ranges of $Y$-valued measures if and only if its dual operator $T^{*}$ factors through a subspace of an $L^{1}(\mu)$-space. In fact, we prove that every compact is taken into a subset of a compact range. We also prove that $T^{*}$ is 1 -summing if and only if $T$ maps compact subsets into subsets of $Y$ lying inside ranges of vector measures with bounded variation.
\end{abstract}

\section{INTRODUCTION}

In [PR] the authors proved that the compact subsets of a Banach space $X$ lie inside ranges of $X$-valued measures if and only if $X^{*}$ can be embedded in an $L^{1}$-space. In this paper we introduce the operator ideal $\mathscr{R}$ of linear operators taking compact subsets of $X$ into subsets that lie inside the range of a $Y$-valued measure. Another natural operator ideal arises if we require these measures to have bounded variation. So we denote by $\mathscr{R}_{\mathrm{bv}}$ the operator ideal of linear operators taking compact subsets of $X$ into subsets that lie inside the range of a $Y$-valued measure with bounded variation.

The study of the ideal $\mathscr{R}$ (resp., $\mathscr{R}_{\mathrm{bv}}$ ) is simplified thanks to the fact that in order for every compact subset of $X$ to lie inside the range of an $X$-valued measure (resp. with bounded variation) it is enough to check norm null sequences. This is based on the fact that every norm compact set is contained inside the closed convex hull of a norm null sequence and, on the other hand, on an important stability result concerning vector measures: if $K$ is the range of a vector measure, so is $\overline{\mathrm{co}}(K)$.

In $\S 1$ we introduce the notation and recall some results about ranges of vector measures. In $\S 2$ we study the bounded variation case and we show that $\mathscr{R}_{\mathrm{bv}}$ is the dual ideal of $\Pi_{1}$. In $\S 3$ we study the ideal $\mathscr{R}$ and prove that $T$ belongs to $\mathscr{R}$ if and only if $T^{*}$ factors through a subspace of an $L^{1}(\mu)$-space. We complete this section showing that an operator $T$ belongs to $\mathscr{R}$ if and only if $S \circ T$ belongs to $\mathscr{R}_{\mathrm{bv}}$ whenever $S$ is 1-summing.

Received by the editors April 18, 1991.

1991 Mathematics Subject Classification. Primary 46G10; Secondary 47B10. 


\section{NOTATION AND PRELIMINARIES}

We will use the classical notation in Banach space theory. We will consider all Banach space over real numbers. If $X$ is a Banach space, $X^{*}$ will denote its dual space and $B_{X}$ its closed unit ball. For a subset $K$ of $X, \overline{\mathrm{co}}(K)$ will be the closed convex hull of $K$. As usual $c_{0}(X) \quad\left(l_{1}(X)\right)$ will stand for the Banach space of norm null sequences (absolutely convergent series) in $X$.

If $T: X \rightarrow Y$ is a continuous and linear operator, we shorten to operator. We refer to $[\mathrm{P}]$ or $[\mathrm{J}]$ for the definition of the nuclear and $p$-summing norm $(1 \leq p<\infty)$ of an operator $T$, denoted respectively by $n(T)$ and $\pi_{p}(T)$. If $X$ and $Y$ are Banach spaces, $\mathscr{N}(X, Y)\left(\Pi_{p}(X, Y)\right)$ will be the space of nuclear ( $p$-summing) operators from $X$ into $Y$.

We will consider only countably additive measures defined on $\sigma$-algebras. If $\Sigma$ is a $\sigma$-algebra of subsets of a set $\Omega, X$ is a Banach space, and $m: \Sigma \rightarrow X$ is such a measure, we will denote by $|m|$ the variation of $m$, which is an extended positive measure, by $\operatorname{tv}(m)$ its total variation, that is, $\operatorname{tv}(m)=|m|(\Omega)$, and by $\|m\|$ its (total) semivariation

$$
\|m\|=\sup \left\{\left|x^{*} \circ m\right|(\Omega): x^{*} \in B_{X^{*}}\right\} .
$$

If $\operatorname{tv}(m)<+\infty$, we will say that $m$ has bounded variation. The range of $m$ will be denoted by $\operatorname{rg}(m)$, that is, $\operatorname{rg}(m)=\{m(A): A \in \Sigma\}$.

We will need several known results providing us the existence of sufficiently many ranges of measures. We summarize them in the next three propositions.

Proposition A. If $X$ is a Banach space and $m$ is an $X$-valued measure, then there exists an $X$-valued measure $\bar{m}$ such that $\overline{\operatorname{co}}(\operatorname{rg}(m))=\operatorname{rg}(\bar{m})$. In this case, we have $\|m\|=\|\bar{m}\|$ and $\operatorname{tv}(m)=\operatorname{tv}(\bar{m})$.

The existence of $\bar{m}$ is due to Ryll-Nardzewsky, see [Du, p. 274]. For the last assertion see $[R]$.

The next proposition can be proved easily using a direct sum of vector measures [KK, p. 35].

Proposition B. Let $X$ be a Banach space and $\left(m_{n}\right)$ a sequence of $X$-valued measures. If $\sum\left\|m_{n}\right\|<+\infty$ (resp. $\left.\sum \operatorname{tv}\left(m_{n}\right)<+\infty\right)$, then there exists an $X$-valued measure in $m$ (resp. of bounded variation) such that

(a) $\operatorname{rg}(m)=\sum \operatorname{rg}\left(m_{n}\right)=\left\{\sum_{n=1}^{\infty} x_{n}: x_{n} \in \operatorname{rg}\left(m_{n}\right)\right\}$,

(b) $\|m\| \leq \sum\left\|m_{n}\right\|\left(\operatorname{tv}(m)=\sum \operatorname{tv}\left(m_{n}\right)\right)$.

If $m: \Sigma \rightarrow X$ is a countably additive vector measure then it is well known that there exists a "control measure" for $m$, that is, a nonnegative countably additive measure $\mu$ so that $m$ is $\mu$-continuous. The linear bounded operator $T_{m}: f \in L^{\infty}(\mu) \rightarrow \int f d m \in X$ is weak*-weak continuous. As was mentioned earlier, $\overline{\operatorname{co}}(\operatorname{rg}(m))$ is also the range of a vector measure. In fact, based on Ryll-Nardzewsky's result, we have

Proposition C. Let $m: \Sigma \rightarrow X$ be a vector measure and $\mu$ a control measure for $m$. Then there exists an $X$-valued measure $\widetilde{m}$ such that $\operatorname{rg}(\widetilde{m})=T_{m}\left(B_{L^{\infty}(\mu)}\right)$. If $m$ has bounded variation then $\tilde{m}$ can be chosen so that $\operatorname{tv}(\tilde{m}) \leq 2 \operatorname{tv}(m)$. 
Proof. Let $\mathscr{B}$ be the Borel $\sigma$-algebra of subsets of $[-1,1]$ and $\lambda$ the Lebesgue measure on $[-1,1]$. Let $\widetilde{\Omega}=\Omega \times[-1,1]$ and $\widetilde{\Sigma}$ be the $\sigma$-algebra generated by $\Sigma \times \mathscr{B}$. Define $\tilde{m}: \widetilde{\Sigma} \rightarrow X$ by

$\widetilde{m}(\widetilde{E})=\int_{\Omega} \lambda(\{t \in[0,1]:(\omega, t) \in \widetilde{E}\}) d m-\int_{\Omega} \lambda(\{t \in[-1,0]:(\omega, t) \in \widetilde{E}\}) d m$

for all $\widetilde{E} \in \widetilde{\Sigma}$. Obviously, $\operatorname{rg}(\widetilde{m}) \subset T_{m}\left(B_{L^{\infty}(\mu)}\right)$. To check the reverse inclusion, let $f \in B_{L^{\infty}(\mu)}$. Choose $g: \Omega \rightarrow \mathbb{R}$ measurable such that $g=f$ $\mu$-almost everywhere and $\|g\|_{\infty}=\|f\|_{\infty}$. Then it is sufficient to look at $\widetilde{E}_{+}=$ $\{(\omega, t): 0 \leq t \leq g(\omega)\}$ and $\widetilde{E}_{-}=\{(\omega, t): g(\omega) \leq t<0\}$ to get $\int_{\Omega} f d m=$ $\widetilde{m}\left(\widetilde{E}_{+} \cup \widetilde{E}_{-}\right)$.

\section{OPERATORS WHOSE DUAL IS 1 -SUMMING}

We denote by $R_{\mathrm{bv}}(X)$ the vector space of all sequences $\left(x_{n}\right)$ in $X$ so that there exists an $X$-valued measure $m$ with finite variation and satisfying

$$
\left\{x_{n}: r_{i} \in \mathbb{N}\right\} \subset \operatorname{rg}(m) \text {. }
$$

If $\left(x_{n}\right)$ belongs to $R_{\mathrm{bv}}(X)$ we put $\left\|\left(x_{n}\right)\right\|_{\mathrm{bv}}=\inf \operatorname{tv}(m)$, where the infimum is taken over all vector measures $m$ admissible in (1). Obviously, we have

$$
\left\|\left(x_{n}\right)\right\|_{\infty} \leq\left\|\left(x_{n}\right)\right\|_{\mathrm{bv}} \text { for all }\left(x_{n}\right) \in R_{\mathrm{bv}}(X) .
$$

We also consider the space $R_{\mathrm{bvc}}(X)$ of all sequences $\left(x_{n}\right)$ in $X$ such that there exists an absolutely convergent series $\sum y_{n}$ satisfying

$$
\left\{x_{n}: n \in \mathbb{N}\right\} \subset \sum_{n=1}^{\infty}\left[-y_{n}, y_{n}\right] \text {, }
$$

where

$$
\sum_{n=1}^{\infty}\left[-y_{n}, y_{n}\right]=\left\{\sum \alpha_{n} y_{n}:\left(\alpha_{n}\right) \in l_{\infty},\left\|\left(\alpha_{n}\right)\right\|_{\infty} \leq 1\right\} .
$$

$\sum_{n=1}^{\infty}\left[-y_{n}, y_{n}\right]$ is called a countable sum of segments; it is a compact convex subset of $X$. The operator $T: l_{\infty} \rightarrow X$ defined by $T\left(\alpha_{n}\right)=\sum \alpha_{n} y_{n}$ is weak $^{*}$ weak continuous, compact, 1 -summing and we have

$$
\sum_{n=1}^{\infty}\left[-y_{n}, y_{n}\right]=T\left(B_{l_{\infty}}\right) \text {. }
$$

$\sum_{n=1}^{\infty}\left[-y_{n}, y_{n}\right]$ is the range of an obvious measure with finite variation equal to $2 \sum\left\|y_{n}\right\|$, so we have $R_{\mathrm{bvc}}(X) \subset R_{\mathrm{bv}}(X)$. If $\left(x_{n}\right)$ belongs to $R_{\mathrm{bvc}}(X)$ we set

$$
\left\|\left(x_{n}\right)\right\|_{\mathrm{bvc}}=\inf \sum_{n=1}^{\infty}\left\|y_{n}\right\|
$$

the infimum being taken over all absolutely convergent series admissible in (3). Obviously, we have

$$
\left\|\left(x_{n}\right)\right\|_{\infty} \leq\left\|\left(x_{n}\right)\right\|_{\mathrm{bv}} \leq 2\left\|\left(x_{n}\right)\right\|_{\mathrm{bvc}}
$$


Proposition 2.1. (a) $\left(R_{\mathrm{bv}}(X),\|\cdot\|_{\mathrm{bv}}\right)$ and $\left(R_{\mathrm{bvc}}(X),\|\cdot\|_{\mathrm{bvc}}\right)$ are Banach spaces. by

(b) A sequence $\left(x_{n}\right)$ belongs to $R_{\mathrm{bvc}}(X)$ if and only if the linear map defined

$$
\left(\alpha_{n}\right) \in l_{1} \rightarrow \sum_{n=1}^{\infty} \alpha_{n} x_{n} \in X
$$

is nuclear.

(c) If $\sum x_{n}^{*}$ is a weakly unconditionally Cauchy (w.u.C.) series in $X^{*}$, then the linear form defined by

$$
\phi:\left(x_{n}\right) \in R_{\mathrm{bvc}}(X) \rightarrow \sum_{n=1}^{\infty}\left\langle x_{n}, x_{n}^{*}\right\rangle \in \mathbb{R}
$$

is well defined and continuous.

Proof. We omit the straightforward verifications of statements (a) and (b) and only make a remark on the completeness assertion. To this end, it suffices to prove that any absolutely summable series in $R_{\mathrm{bv}}(X)$ (resp. $\left.R_{\mathrm{bvc}}(X)\right)$ is convergent. In the case of $R_{\mathrm{bv}}(X)$ we will use Proposition B.

(c) If $\left(x_{n}\right)$ is a sequence belonging to $R_{\mathrm{bvc}}(X)$ and we choose an absolutely convergent series $\sum y_{n}$ so that

$$
\left\{x_{n}: n \in \mathbb{N}\right\} \subset\left\{\sum_{k=1}^{\infty} \alpha_{k} y_{k}:\left|\alpha_{k}\right| \leq 1\right\},
$$

then we have

$$
\sum_{n=1}^{\infty}\left|\left\langle x_{n}, x_{n}^{*}\right\rangle\right| \leq \sum_{k=1}^{\infty} \sum_{n=1}^{\infty}\left|\left\langle y_{k}, x_{n}^{*}\right\rangle\right| \leq\left(\sum_{k=1}^{\infty}\left\|y_{k}\right\|\right) \sup \left\{\sum_{n=1}^{\infty}\left|\left\langle x, x_{n}^{*}\right\rangle\right|:\|x\| \leq 1\right\} .
$$

Then $\phi$ is well defined and continuous since

$$
\left|\left\langle\phi,\left(x_{n}\right)\right\rangle\right| \leq\left(\sup \left\{\sum_{n=1}^{\infty}\left|\left\langle x, x_{n}^{*}\right\rangle\right|:\|x\| \leq 1\right\}\right)\left\|\left(x_{n}\right)\right\|_{\text {bvc }} .
$$

Theorem 2.2. Let $T$ be a continuous and linear map from $X$ into $Y$. The following statements are equivalent:

(a) $\left(T x_{n}\right)$ belongs to $R_{\mathrm{bv}}(Y)$ for every null sequence $\left(x_{n}\right)$ in $X$.

(b) There exists a constant $c>0$ such that for every finite subset $H$ of $B_{X}$ there is a $Y$-valued measure $m$ so that $T(H) \subset \operatorname{rg}(m)$ and $\operatorname{tv}(m) \leq c$.

(c) There exists a constant $c>0$ such that for every finite subset $H$ of $B_{X}$ there is a finite subset $\left\{y_{j}\right\}_{j=1}^{m}$ of $Y$ so that $T(H) \subset \sum_{j=1}^{m}\left[-y_{j}, y_{j}\right]$ and $\sum_{j=1}^{m}\left\|y_{j}\right\| \leq c$.

(d) $\left(T x_{n}\right)$ belongs to $R_{\mathrm{bvc}}(Y)$ for every null sequence $\left(x_{n}\right)$ in $X$.

Proof. $(\mathrm{a}) \Rightarrow(\mathrm{b})$ Define $\widehat{T}: c_{0}(X) \rightarrow R_{\mathrm{bv}}(Y)$ by

$$
\left(x_{n}\right) \in c_{0}(X) \rightarrow\left(T x_{n}\right) \in R_{\mathrm{bv}}(Y) .
$$

Then $\widehat{T}$ is linear and has closed graph; therefore, $\widehat{T}$ is bounded. So we have

$$
\left\|\left(T x_{n}\right)\right\|_{r} \leq\|\widehat{T}\|\left\|\left(x_{n}\right)\right\|_{\infty}
$$

for all $\left(x_{n}\right) \in c_{0}(X)$ and (b) follows with $c=\|\widehat{T}\|$. 
(b) $\Rightarrow$ (c) Let $H=\left\{x_{1}, \ldots, x_{n}\right\}$ be a finite subset of $B_{X}$. There exists a $Y$ valued measure $m$ such that $\operatorname{tv}(m) \leq c$ and $T(H) \subset \operatorname{rg}(m)$. Choose $A_{i}$ such that $T x_{i}=m\left(A_{i}\right)$ and consider the $\sigma$-field $\Sigma_{0}$ generated by $\left\{A_{1}, \ldots, A_{n}\right\}$ on $\Omega$. If $E_{1}, \ldots, E_{m}$ are the atoms of $\Sigma_{0}$, we put $y_{j}=m\left(E_{j}\right)$ for $j=1, \ldots, m$. Let us note that

$$
T(H) \subset\left\{\sum_{j=1}^{m} \alpha_{j} y_{j}:\left|\alpha_{j}\right| \leq 1\right\}
$$

and

$$
\sum_{j=1}^{m}\left\|y_{j}\right\| \leq|m|\left(\bigcup_{j=1}^{m} E_{j}\right) \leq \operatorname{tv}(m) \leq c .
$$

Hence we get (c).

(c) $\Rightarrow(\mathrm{d})$ Define $\widehat{T}: X^{(N)} \rightarrow Y^{(N)}$ by

$$
\left(x_{n}\right) \in\left(X^{(N)},\|\cdot\|_{\infty}\right) \rightarrow\left(T x_{n}\right) \in\left(Y^{(N)},\|\cdot\|_{\mathrm{bvc}}\right),
$$

where $X^{(N)}$ denotes the vector space of all sequences $\left(x_{n}\right)$ is $X$ that contain only finitely many $x_{n} \neq 0$. Then $\widehat{T}$ is linear and such that $\left\|\widehat{T}\left(x_{n}\right)\right\|_{\text {bvc }} \leq$ $c\left\|\left(x_{n}\right)\right\|_{\infty}$ for all $\left(x_{n}\right) \in X^{(N)}$. Since $X^{(N)}$ is dense in $c_{0}(X)$, using (4), it follows that $\left(T x_{n}\right)$ belongs to $R_{\mathrm{bvc}}(Y)$ for all null sequences $\left(x_{n}\right)$ in $X$ and $\left\|\left(T x_{n}\right)\right\|_{\text {bvc }} \leq c\left\|\left(x_{n}\right)\right\|_{\infty}$.

(d) $\Rightarrow$ (a) This is obvious.

Let $X$ and $Y$ be real Banach spaces. We say that an operator $T: X \rightarrow Y$ belongs to $\mathscr{R}_{\mathrm{bv}}(X, Y)$ if it takes each null sequence $\left(x_{n}\right)$ in $X$ into a sequence $\left(T x_{n}\right)$ lying inside the range of a $Y$-valued measure with finite variation. It can easily be seen that $\mathscr{R}_{\mathrm{bv}}$ is an operator ideal in the sense of Pietsch. We will prove that $\mathscr{R}_{\mathrm{bv}}$ is the dual operator ideal of $\Pi_{1}$ (see $\left.[\mathrm{P}]\right)$.

We suppose $Y$ is identified with a subspace of $Y^{* *}$ and denote the canonical injection from $Y$ into $Y^{* *}$ by $J$. Then we have

Proposition 2.3. If $T$ is a continuous and linear operator from $X$ into $Y$, then $T \in \mathscr{R}_{\mathrm{bv}}(X, Y)$ if and only if $J T \in \mathscr{R}_{\mathrm{bv}}\left(X, Y^{* *}\right)$.

Proof. The "if" part is obvious since $\mathscr{R}_{\mathrm{bv}}$ is an operator ideal. To prove the "only if" part, let $T$ be an operator such that $J T \in \mathscr{R}_{\mathrm{bv}}\left(X, Y^{* *}\right)$. By Theorem 1.2 , there exists a constant $c>0$ so that every finite subset $H=\left\{x_{i}\right\}_{i=1}^{n}$ of $B_{X}$ lies inside the range of a $Y^{* *}$-valued measure $m$ for which $\operatorname{tv}(m) \leq c$. Choose $A_{i}$ so that $J T x_{i}=m\left(A_{i}\right)$ and consider the $\sigma$-algebra $\Sigma_{0}$ generated by $\left\{A_{1}, \ldots, A_{n}\right\}$ on $\Omega$. Let $\tilde{m}$ be the restriction of $m$ to $\Sigma_{0}$ and $Z$ be the finitedimensional linear span of $\operatorname{rg}(\tilde{m})$. By the "principle of Local Reflexivity" [Pi, p. 6], given $\varepsilon>0$, there exists a one-to-one operator $S: Z \rightarrow Y$, with $S x=x$ for all $x \in Z \cap Y$, and $\|S\| \leq 1+\varepsilon$. Thus $m_{1}=S \circ \tilde{m}$ is a $Y$-valued measure for which we have $\left\{T x_{i}\right\}_{i=1}^{n} \subset \operatorname{rg}\left(m_{1}\right)$ and $\operatorname{tv}\left(m_{1}\right) \leq(1+\varepsilon) c$. The proposition follows from Theorem 2.2.

Theorem 2.4. If $X$ and $Y$ are real Banach spaces, the following statements are equivalent:

(a) $T \in \mathscr{R}_{\mathrm{bv}}(X, Y)$,

(b) $T^{*}: Y^{*} \rightarrow X^{*}$ is 1-summing. 
Proof. (a) $\Rightarrow$ (b) Let $\sum y_{n}^{*}$ be a w.u. Cauchy series in $Y^{*}$. By Theorem 2.2, the linear map $\widehat{T}$ defined by

$$
\left(x_{n}\right) \in c_{0}(X) \rightarrow\left(T x_{n}\right) \in R_{\mathrm{bvc}}(Y)
$$

is continuous. Since Proposition 1.1 tells us that the linear form

$$
\phi:\left(y_{n}\right) \in R_{\mathrm{bvc}}(Y) \rightarrow \sum\left\langle y_{n}, y_{n}^{*}\right\rangle \in \mathbb{R}
$$

is continuous, it follows that $\phi \circ \widehat{T}$ is a linear and continuous form on $c_{0}(X)$. Thus $\sum\left\|T^{*} y_{n}^{*}\right\|<+\infty$ because $l_{1}\left(X^{*}\right)$ is the dual space of $c_{0}(X)$.

(b) $\Rightarrow$ (a) If $\left(x_{n}\right)$ is a null sequence in $X$, we consider the linear map $A$ defined by $\left(\alpha_{n}\right) \in l_{1} \rightarrow \sum \alpha_{n} T x_{n} \in Y$. Its dual map $A^{*}: y^{*} \in Y^{*} \rightarrow$ $\left(\left\langle T x_{n}, y^{*}\right\rangle\right) \in l_{\infty}$ is 1 -summing because it is the composition of the maps $x_{*} \in$ $X^{*} \rightarrow\left(\left\langle x_{n}, x_{*}\right\rangle\right) \in l_{\infty}$ and $T^{*}$. Then $A^{*}$ is integral and, hence, so is $A$. By [DU, p. 234], $A$ admits a factorization

$$
\begin{aligned}
l_{1} \rightarrow & Y \underset{Y^{* *}}{\rightarrow} \underset{L^{\infty}(\mu)}{\longrightarrow} \rightarrow L^{1}(\mu)
\end{aligned}
$$

where $\mu$ is a finite regular measure on some compact Hausdorff space $\Omega$, $I: L^{\infty}(\mu) \rightarrow L^{1}(\mu)$ is the natural inclusion, and $S$ and $Q$ are bounded linear operators. Since $I$ is weak*-weak continuous and 1 -summing, it has a representing measure $m$ with bounded variation. It follows from Proposition $C$ that there is an $L^{1}(\mu)$-valued measure $\widetilde{m}$ with bounded variation such that $\operatorname{rg}(\tilde{m})=I\left(B_{L^{\infty}(\mu)}\right)$. Then $m_{1}=Q \circ \widetilde{m}$ is a $Y^{* *}$-valued measure so that $J T x_{n} \in \alpha \operatorname{rg}\left(m_{1}\right)$ for all $n \in \mathbb{N}$ and a suitable constant $\alpha>0$. This proves that $J T \in \mathscr{R}_{\mathrm{bv}}\left(X, Y^{* *}\right)$. A look at Proposition 2.3 will tell you that $T \in \mathscr{R}_{\mathrm{bv}}(X, Y)$.

\section{THE OPERATOR IDEAL $\mathscr{R}$}

In [PR] the authors introduced the vector space $R(X)$ of all sequences $\left(x_{n}\right)$ in $X$ so that there exists an $X$-valued measure $m$ satisfying

$$
\left\{x_{n}: n \in \mathbb{N}\right\} \subset \operatorname{rg}(m),
$$

endowed with the norm $\left\|\left(x_{n}\right)\right\|_{r}=$ inf $\|m\|$, where the infimum is taken over all vector measures $m$ admissible in (5).

We also consider sequences in $X$ that lie inside the range of a vector measure with relatively compact range. We denote by $R_{c}(X)$ the vector space of all such sequences $\left(x_{n}\right)$ in $X$. By [PR, Proposition 1.4], if $\left(x_{n}\right)$ belongs to $R_{c}(X)$, there exists an unconditionally convergent series $\sum y_{k}$ satisfying $x_{n} \in$ $\sum\left[-y_{k}, y_{k}\right]$ for all $n$. The operator $T: l_{\infty} \rightarrow X$ defined by $T\left(\alpha_{n}\right)=\sum \alpha_{n} y_{n}$ is weak ${ }^{*}$-weak continuous, compact and we have

$$
\sum_{n=1}^{\infty}\left[-y_{n}, y_{n}\right]=T\left(B_{l_{\infty}}\right)
$$

By Proposition C, $\sum\left[-y_{n}, y_{n}\right]$ is the range of a measure $m$ with compact range for which

$$
\|m\| \leq 2 \sup \left\{\sum_{k=1}^{\infty}\left|\left\langle y_{k}, x^{*}\right\rangle\right|: x^{*} \in B_{X^{*}}\right\} .
$$


If $\left(x_{n}\right)$ belongs to $R_{c}(X)$ we set

$$
\left\|\left(x_{n}\right)\right\|_{r c}=\inf \sup \left\{\sum_{k=1}^{\infty}\left|\left\langle y_{k}, x^{*}\right\rangle\right|: x^{*} \in B_{X^{*}}\right\},
$$

the infimum being taken over all u.c. series $\sum y_{k}$ such that $\left\{x_{n}\right\}$ is contained in $\sum\left[-y_{k}, y_{k}\right]$.

The next proposition contains without proof some properties of the spaces $R(X)$ and $R_{c}(X)$, which we will need (see [PR]).

Proposition 3.1. (a) $\left(R(X),\|\|_{r}\right)$ and $\left(R_{c}(X),\|\|_{r c}\right)$ are Banach spaces.

(b) $\left\|\left(x_{n}\right)\right\|_{\infty} \leq\left\|\left(x_{n}\right)\right\|_{r} \leq 2\left\|\left(x_{n}\right)\right\|_{r c}$ for all $\left(x_{n}\right) \in R_{c}(X)$.

(c) $\left\|\left(x_{n}\right)\right\|_{\infty} \leq\left\|\left(x_{n}\right)\right\|_{r}$ for all $\left(x_{n}\right) \in R(X)$.

(d) If $c_{0}(X) \subset R(X)$ then $c_{0}(Z) \subset R(Z)$ for every quotient $Z$ of $X$.

(e) $c_{0}\left(L^{\infty}(\lambda)\right) \subset R\left(L^{\infty}(\lambda)\right)$ for every extended positive measure.

Using a similar argument to that of the bounded variation case, we have

Proposition 3.2. If $T$ is a continuous and linear operator from $X$ into $Y$, then $T \in \mathscr{R}(X, Y)$ if and only if $J T \in \mathscr{R}\left(X, Y^{* *}\right)$.

Theorem 3.3. Let $T$ be an operator from $X$ into $Y$. The following statements are equivalent:

(a) $\left(T x_{n}\right)$ belongs to $R(Y)$ for every null sequence $\left(x_{n}\right)$ in $X$.

(b) There exists a constant $c>0$ such that for every finite subset $H$ of $B_{X}$ there is a $Y$-valued measure $m$ so that $T(H) \subset \operatorname{rg}(m)$ and $\|m\| \leq c$.

(c) There exists a constant $c>0$ such that for every finite subset $H$ of $B_{X}$ there is a finite subset $\left\{y_{j}\right\}_{j=1}^{m}$ of $Y$ so that $T(H) \subset \sum_{j=1}^{m}\left[-y_{j}, y_{j}\right]$ and

$$
\sup \left\{\sum_{j=1}^{m}\left|\left\langle y_{j}, y^{*}\right\rangle\right|: y^{*} \in B_{X^{*}}\right\} \leq c .
$$

(d) $\left(T x_{n}\right)$ belongs to $R_{c}(Y)$ for every null sequence $\left(x_{n}\right)$ in $X$.

(e) There exists a constant $c>0$ such that for every finite-dimensional subspace $E$ of $Y^{*}$ the operator $T_{E}^{*}: E \rightarrow X^{*}$ admits a factorization

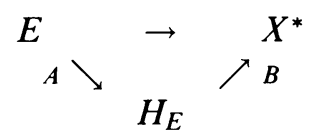

where $H_{E}$ is a subspace of $l_{1},\|A\| \leq 1$, and $\|B\| \leq c$.

(f) $T^{*}$ factors through a subspace $H$ of an $L^{1}(\mu)$-space.

Proof. The proofs of $(\mathrm{a}) \Rightarrow(\mathrm{b}),(\mathrm{b}) \Rightarrow(\mathrm{c})$, and $(\mathrm{c}) \Rightarrow(\mathrm{d})$ are similar to that of the bounded variation case.

(d) $\Rightarrow$ (e) Let $E$ be a finite-dimensional subspace of $Y^{*}$. Given $\varepsilon>0$, we choose a finite subset $\left\{x_{1}, \ldots, x_{n}\right\}$ of $S_{X}$ so that

$$
\left\|x^{*}\right\| \leq(1+\varepsilon) \sup \left\{\left|\left\langle x_{i}, x^{*}\right\rangle\right|: i=1, \ldots, n\right\} \quad \text { for all } x^{*} \in T^{*}(E) .
$$

By (d) there exists an unconditionally convergent series $\sum y_{n}$ in $Y$ such that

$$
\left\{T x_{i}: i \leq n\right\} \subset \sum_{j=1}^{\infty}\left[-y_{j}, y_{j}\right]
$$


and

$$
\sup \left\{\sum_{j=1}^{\infty}\left|\left\langle y_{j}, y^{*}\right\rangle\right|: y^{*} \in B_{Y^{*}}\right\} \leq c .
$$

We now define $A: E \rightarrow l_{1}$ by $y^{*} \in E \rightarrow\left(c^{-1}\right) \sum\left\langle y_{j}, y^{*}\right\rangle e_{j} \in l_{1}$ and $B: A(E) \rightarrow X^{*}$ by $B\left(A y^{*}\right)=T^{*} y^{*}$. To prove that $B$ is well defined, let $y^{*} \in E$ such that $A y^{*}=0$. Then $\left\langle y_{j}, y^{*}\right\rangle=0$ for all $j \in \mathbb{N}$, and it follows from (7) that $\left\langle T x_{i}, y^{*}\right\rangle=0$ for $i=1, \ldots, n$. Thus (6) yields $T^{*} y^{*}=0$.

Finally, by (7) and (8) we have $\|A\| \leq 1$ and $\|B\| \leq(1+\varepsilon) c$.

(e) $\Rightarrow$ (f) By a similar argument to that in [LP, Proposition 7.1] one can prove that $T^{*}$ admits a factorization through a subspace of an $L^{1}(\mu)$-space.

(f) $\Rightarrow$ (a) If $T^{*}$ admits such a factorization, then $T^{* *}$ factors through a quotient of an $L^{\infty}(\mu)$-space. By Proposition 3.1, $T$ maps each null sequence $\left(x_{n}\right)$ into a sequence $\left(T x_{n}\right)$ lying inside the range of a $Y^{* *}$-valued measure. Then Proposition 3.2 yields $T \in \mathscr{R}(X, Y)$.

Finally, we have obtained the following multiplication theorem.

Theorem 3.4. Let $T$ be an operator from $X$ into $Y$. The following statements are equivalent:

(a) $T \in \mathscr{R}(X, y)$.

(b) $S T \in \mathscr{R}_{\mathrm{bv}}(X, Z)$ for every Banach space $Z$ and every operator $S \in$ $\Pi_{1}(Y, Z)$.

(c) $S T \in N\left(X, l_{1}\right)$ for every operator $S \in \Pi_{1}\left(Y, l_{1}\right)$.

(d) There exists a constant $c>0$ such that $\sum_{i=1}^{n}\left\|T^{*} y_{i}^{*}\right\| \leq c \sum_{j=1}^{m}\left\|y_{j}^{*}\right\|$ whenever $\sum_{i=1}^{n}\left|\left\langle y, y_{i}^{*}\right\rangle\right| \leq \sum_{j=1}^{m}\left|\left\langle y, y_{j}^{*}\right\rangle\right|$ for all $y \in Y$.

Proof. (a) $\Rightarrow$ (b) Let $\left(x_{n}\right)$ be a null sequence in $X$. By Theorem 3.3 there exists an unconditionally convergent series $\sum y_{n}$ in $Y$ so that $T x_{n} \in$ $\sum_{j=1}^{\infty}\left[-y_{j}, y_{j}\right]$ for all $n \in \mathbb{N}$. Then $S T x_{n} \in \sum_{j=1}^{\infty}\left[-S y_{j}, S y_{j}\right]$ for all $n \in \mathbb{N}$ and $\sum\left\|S y_{j}\right\|<+\infty$. So $\left(S T x_{n}\right)$ belongs to $R_{\mathrm{bvc}}(Z)$.

(b) $\Rightarrow$ (c) Let $x_{n}^{*}=T^{*} S^{*}\left(e_{n}\right)$. By Theorem 2.2, $(S T)^{*}: l_{\infty} \rightarrow X^{*}$ is 1summing, so $\sum x_{n}^{*}$ is an absolutely convergent series. Then $S T x=\sum\left\langle x, x_{n}^{*}\right\rangle e_{n}$, so $S T$ is nuclear.

(c) $\Rightarrow$ (d) Since $S T$ belongs to $\mathscr{N}\left(X, l_{1}\right)$ for all $S \in \Pi_{1}\left(Y, l_{1}\right)$, we can define the operator $U: S \in \Pi_{1}\left(Y, l_{1}\right) \rightarrow S T \in \mathscr{N}\left(X, l_{1}\right)$ that has closed graph. Indeed, if $\left(S_{n}, S_{n} T_{n}\right)_{n}$ is convergent to $(S, R)$ in $\Pi_{1}\left(Y, l_{1}\right) \times \mathscr{N}\left(X, l_{1}\right)$, then $S_{n} \rightarrow S$ in $\Pi_{1}\left(Y, l_{1}\right)$ and $S_{n} T_{n} \rightarrow R$ in $\mathscr{N}\left(X, l_{1}\right)$. Since $\pi_{1}(A) \leq n(A)$ for all nuclear operator, it follows that

$$
\begin{aligned}
\pi_{1}(R-S T) & \leq \pi_{1}\left(R-S_{n} T\right)+\pi_{1}\left(S_{n} T-S T\right) \\
& \leq n\left(R-S_{n} T\right)+\|T\| \pi_{1}\left(S_{n}-S\right) .
\end{aligned}
$$

This yields immediately that $R-S T=0$, so $U$ has closed graph. Hence there is a constant $c>0$ such that

$$
n(S T) \leq c \pi_{1}(S) \text { for all } S \in \Pi_{1}\left(Y, l_{1}\right) .
$$

If $\left\{y_{i}^{*}\right\}_{i=1}^{n}$ and $\left\{y_{j}^{*}\right\}_{j=1}^{m}$ are finite subsets of $Y^{*}$ so that

$$
\sum_{i=1}^{n}\left|\left\langle y, y_{i}^{*}\right\rangle\right| \leq \sum_{j=1}^{m}\left|\left\langle y, y_{j}^{*}\right\rangle\right| \quad \text { for all } y \in Y,
$$


we define two linear operators $A$ and $B$ from $Y$ into $l_{1}$ by

$$
A y=\sum\left\langle y, y_{i}^{*}\right\rangle e_{i} \text { and } B y=\sum\left\langle y, y_{j}^{*}\right\rangle e_{j}
$$

for all $y \in Y$, where $\left\{e_{n}: n \in \mathbb{N}\right\}$ is the unit basis of $l_{1}$. From (10) it follows that $\pi_{1}(A) \leq \pi_{1}(B)$. This and (9) yield

$$
n(A T) \leq c \pi_{1}(A) \leq c \pi_{1}(B) \leq c n(B) .
$$

But $n(A T)=\sum\left\|T^{*} y_{i}^{*}\right\|$ and $n(B)=\sum\left\|y_{j}^{*}\right\|$ because the Banach spaces $\mathscr{N}\left(X, l_{1}\right)$ and $l_{1}\left(X^{*}\right)$ are isometric [J, 1.15]. So the implication follows.

(d) $\Rightarrow$ (a) We shall show that for every finite-dimensional subspace $E$ of $Y^{*}$ the operator $T_{E}^{*}: E \rightarrow X^{*}$ admits a factorization

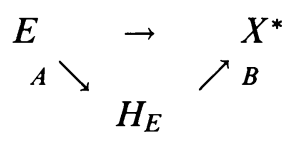

where $H_{E}$ is a subspace of an $L^{1}(\mu)$-space, $\|A\| \leq 1$, and $\|B\| \leq c$. Then (a) follows from Theorem 3.3. To prove that $T_{E}^{*}$ admits such a factorization, using a similar argument given in the proof of [PL, Theorem 7.3], one can prove that there exists a positive measure $\mu$ on $S=\left\{s \in E^{*}:\|s\|=1\right\}$ such that

$$
\int_{S}\left|\left\langle z^{*}, s\right\rangle\right| d \mu \leq c \int_{S}\left|\left\langle y^{*}, s\right\rangle\right| d \mu
$$

for all $z^{*} \in E$ and for all $y^{*} \in E$ with $\left\|z^{*}\right\|=\left\|T^{*} y^{*}\right\|=1$. Let

$$
\gamma=\inf \left\{\int_{S}\left|\left\langle y^{*}, s\right\rangle\right| d \mu:\left\|T^{*} y^{*}\right\|=1, y^{*} \in E\right\} .
$$

The number $\gamma$ is not 0 and we can define two operators $A: E \rightarrow L^{1}(\mu)$ by $A y^{*}=(\gamma c)^{-1} \widehat{f_{y^{*}}}$ and $B: A(E) \rightarrow X^{*}$ by $B\left(f_{y^{*}}\right)=T^{*} y^{*}$ satisfying $\|A\| \leq 1$, $\|B\| \leq c$, and $B A=T_{E}^{*}$.

\section{REMARKS AND EXAMPLES}

Example 4.1. Let $1 \leq p \leq 2$. If $T$ is an operator from $X$ into $Y$ such that $T^{*}$ is $p$-summing, then $T \in \mathscr{R}(X, Y)$. Indeed, $T^{*}$ admits a factorization through a subspace of an $L^{p}(\mu)$-space, and it is known that $L^{p}(\mu)$ embeds isometrically into $L^{1}$ for $1 \leq p \leq 2$. However, if $p>2$, there are operators $T$ whose dual $T^{*}$ is $p$-summing but they do not belong to $\mathscr{R}$. In fact, let $p>2$ and choose $c \in(1, p / 2)$. Then the operator $T$ defined by

$$
\left(\alpha_{n}\right) \in l_{q} \rightarrow\left(n^{-c / p} \alpha_{n}\right) \in l_{1}
$$

maps the null sequence $\left(n^{c / p-1 / 2} e_{n}\right)$ into the sequence $\left(e_{n} / \sqrt{n}\right)$ that is not inside the range of a measure [AD], but its dual $T^{*}$ is $p$-summing [P, p. 234].

In Theorem 2.2 we have proved that for an operator $T$ the statement “ $T$ maps each null sequence into a sequence lying inside the range of a vector measure with bounded variation" and " $T$ maps each null sequence into a sequence lying inside a countable sum of segments" are equivalent. However, the next example shows that this equivalence is not true for isolated null sequences. 
Example 4.2. There exists a null sequence in $L^{1}[0,1]$ that lies in the range of a measure with bounded variation but not in any countable sum of segments.

Indeed, the sequence $\left(\chi_{[(k-1) / n, k / n]}\right)_{n \in \mathbb{N}}, 1 \leq k \leq n$ is a null sequence in $L^{1}[0,1]$ that is contained in the range of the vector measure $m: A \in \mathscr{L} \rightarrow \chi_{A} \in L^{1}[0,1]$ where $\mathscr{L}$ denotes the $\sigma$-algebra of Lebesgue measurable subsets of $[0,1]$. But it is not contained in any countable sum of segments. Otherwise, by Proposition 2.1 the operator $\left(\alpha_{n, k}\right) \in l_{1} \rightarrow \sum \alpha_{n, k} \chi_{[(k-1) / n, k / n]} \in L^{1}[0,1]$ would be nuclear. Then, by the well-known Grothendieck Theorem [DU, p. 259], the set $\left\{\chi_{[(k-1) / n, k / n]}: n \in \mathbb{N}, 1 \leq k \leq n\right\}$ ought to be equimeasurable, that is, for every $\varepsilon>0$ there exists a set $A(\varepsilon) \in \mathscr{L}$ so that $\mu(A(\varepsilon))>1-\varepsilon$ and $\left\{\chi_{[(k-1) / n, k / n] \cap A(\varepsilon)}: n \in \mathbb{N}, 1 \leq k \leq n\right\}$ is relatively compact in $L^{\infty}[0,1]$. But obviously, this is not the case.

Remark 4.3. Since the bidual operator $T^{* *}$ of a 1-summing operator $T$ is also 1-summing, Theorem 2.4 yields the following dual characterization of 1summing operators:

(i) $T$ is 1-summing if and only if $T^{*}$ maps each null sequence from $Y^{*}$ into a sequence from $X^{*}$ lying inside a countable sum of segments.

(ii) $T^{*}$ is 1-summing if and only if $T$ maps each null sequence from $X$ into a sequence from $Y$ lying inside a countable sum of segments.

\section{REFERENCES}

[AD] R. Anantharaman and J. Diestel, Sequences in the range of a vector measure, preprint.

[D] J. Diestel, Sequences and series in Banach spaces, Graduate Texts in Math., vol. 92, SpringerVerlag, 1984.

[DU] J. Diestel and J. J. Uhl, Vector measures, Math. Surveys Monographs, vol. 15, Amer. Math. Soc., Providence, RI, 1977.

[KK] I. Kluvanek and G. Knowles, Vector measures and control systems, North-Holland Math. Stud., vol. 20, North-Holland, Amsterdam, 1976.

[P] A. Pietsch, Operator ideals, North-Holland, Amsterdam, 1980.

[Pi] G. Pisier, Factorization of linear operators and geometry of Banach spaces, CBMS, vol. 60, Amer. Math. Soc., Providence, RI, 1984.

[PR] C. Pineiro and L. Rodriguez-Piazza, Banach spaces in which every compact lies inside the range of a vector measure, Proc. Amer. Math. Soc. 114 (1992), 505-517.

[R] L. Rodriguez-Piazza, The range of a vector measure determines its total variation, Proc. Amer. Math. Soc. 111 (1991), 205-214.

[SR] C. P. Stegall and J. R. Retherford, Fully nuclear and completely nuclear operators with applications to $\mathscr{L}_{\infty}$ - and $\mathscr{L}_{\infty}$-spaces, Trans. Amer. Math. Soc. 163 (1972), 457-492.

Departamento de Análisis Matemático, Facultad de Matemáticas, Universidad de Sevilla, Sevilla, 41080 .Aptdo. 1160 , Spain 